\title{
Methodological considerations for kinematic analysis of upper limbs in healthy and poststroke adults Part II: a systematic review of motion capture systems and kinematic metrics
}

Inês Albuquerque Mesquita, Pedro Filipe Pereira da Fonseca, Ana Rita Vieira Pinheiro, Miguel Fernando Paiva Velhote Correia \& Cláudia Isabel Costa da Silva

To cite this article: Inês Albuquerque Mesquita, Pedro Filipe Pereira da Fonseca, Ana Rita Vieira Pinheiro, Miguel Fernando Paiva Velhote Correia \& Cláudia Isabel Costa da Silva (2019) Methodological considerations for kinematic analysis of upper limbs in healthy and poststroke adults Part II: a systematic review of motion capture systems and kinematic metrics, Topics in Stroke Rehabilitation, 26:6, 464-472, DOI: 10.1080/10749357.2019.1611221

To link to this article: https://doi.org/10.1080/10749357.2019.1611221

Published online: 08 May 2019.

Submit your article to this journal ¿

凹ll Article views: 65

Q View related articles $\asymp$

View Crossmark data 


\title{
Methodological considerations for kinematic analysis of upper limbs in healthy and poststroke adults Part II: a systematic review of motion capture systems and kinematic metrics
}

\author{
Inês Albuquerque Mesquita (10), Pedro Filipe Pereira da Fonseca (10) ${ }^{\mathrm{b}}$, Ana Rita Vieira Pinheiro (1) ${ }^{\mathrm{c} d \mathrm{~d}}$ \\ Miguel Fernando Paiva Velhote Correia (1) ${ }^{\mathrm{e}, \mathrm{f}}$ and Cláudia Isabel Costa da Silva (10 ${ }^{\mathrm{d}}$
}

\begin{abstract}
aDepartment of Functional Sciences and Center for Rehabilitation Research (CIR), School of Health of Polytechnic Institute of Porto (ESS-P.Porto), Porto, Portugal; ' 'LABIOMEP: Porto Biomechanics Laboratory, University of Porto, Porto, Portugal; 'School of Health Sciences, University of Aveiro, Aveiro, Portugal; dDepartment of Physiotherapy and Center for Rehabilitation Research (CIR), School of Health of Polytechnic Institute of Porto (ESS-P.Porto), Porto, Portugal; ' $D$ Department of Electrical and Computer Engineering, Faculty of Engineering of the University of Porto (FEUP), Porto, Portugal; fInstitute for Systems and Computer Engineering, Technology and Science (INESC TEC), Porto, Portugal
\end{abstract}

\begin{abstract}
Background and purpose: To review the methods used to analyze the kinematics of upper limbs (ULs) of healthy and poststroke adults, namely the motion capture systems and kinematic metrics. Summary of review: A database of articles published in the last decade was compiled using the following search terms combinations: ("upper extremity" OR "upper limb" OR arm) AND (kinematic OR motion OR movement) AND (analysis OR assessment OR measurement). The articles included in this review: (1) had the purpose to analyze objectively three-dimension kinematics of ULs, (2) studied functional movements or activities of daily living involving ULs, and (3) studied healthy and/or poststroke adults. Fourteen articles were included (four studied a healthy sample, three analyzed poststroke patients, and seven examined both poststroke and healthy participants).

Conclusion: Most articles used optoelectronic systems with markers; however, the presentation of laboratory and task-specific errors is missing. Markerless systems, used in some studies, seem to be promising alternatives for implementation of kinematic analysis in hospitals and clinics, but the literature proving their validity is scarce. Most articles analyzed "joint kinematics" and "end-point kinematics," mainly related with reaching. The different stroke locations of the samples were not considered in their analysis and only three articles described their psychometric properties.

Implication of key findings: Future research should validate portable motion capture systems, document their specific error at the acquisition place and for the studied task, include grasping and manipulation analysis, and describe psychometric properties.
\end{abstract}

ARTICLE HISTORY

Received 24 December 2018

Accepted 3 March 2019

KEYWORDS

Upper extremity; kinematic assessment; stroke; optoelectronic systems; markerless systems; joint kinematics; end-point kinematics

\section{Introduction}

More than $80 \%$ of stroke patients experience acute sensorimotor dysfunction of the contralesional upper limb (UL), which becomes chronic for more than $40 \%$ of the patients. ${ }^{1}$ Although there seem to be promising approaches to promote ULs recovery after stroke, the quantification of the interventions effectiveness remains limited by the available assessment measures. ${ }^{2}$ Recently, the Stroke Recovery and Rehabilitation Roundtable (SRRR) strongly recommended the inclusion of both core clinical measures and kinematics in poststroke recovery trials. ${ }^{3}$ In clinical setting, UL motor impairment is mainly evaluated by clinical tools such as the Fugl-Meyer Assessment for Upper Extremity ${ }^{4}$ and the Action Research Arm Test ${ }^{5}$ which are based on the examiner's observation. ${ }^{6}$ Though they are both valid instruments, ${ }^{3}$ these clinical measures are strongly influenced by the observer's experience. Moreover, since they focus on task achievement rather than on how tasks are performed, ${ }^{8}$ these tools cannot describe the underlying biomechanical characteristics of motor function deficits and, therefore, cannot differentiate restitution (also known as true recovery) from compensation. ${ }^{3}$ Kinematics' parameters are presented as one of the best ways for this purpose and to improve the understanding about the mechanisms that drive motor recovery. ${ }^{3}$

The kinematic analysis allows an accurate and objective assessment of the ULs motor functions by providing objective and quantitative parameters. ${ }^{6-11}$ However, this requires special equipment ${ }^{11}$ and a more complex identification and interpretation of kinematic metrics, which has led to its use mostly in research setting. ${ }^{11}$

Accuracy, reliability, high signal-to-noise ratio, compactness, and cost are very important features to the kinematic analysis acceptance into routine rehabilitation and to the implementation in clinical setting. ${ }^{8}$ Visual marker-based optoelectronic systems are often considered the gold standard in the kinematic analysis because of their high accuracy and reliability, $8,12,13$ and they are used as reference for comparisons with other techniques. ${ }^{14,15}$ These systems use retro-reflective markers (passive or active) which absolute position is detected by multiple

CONTACT Inês Albuquerque Mesquita inesamesquita@gmail.com Department of Functional Sciences and Center for Rehabilitation Research (CIR), School of Health of Polytechnic Institute of Porto (ESS-P.Porto). Rua Dr. António Bernardino de Almeida, 400, 4200 - 072, Porto, Portugal

๑ 2019 Taylor \& Francis Group, LLC 
video cameras in relation to a reference position. ${ }^{13}$ However, the difficulty in transportation, large set-up volume, and high cost make them impractical and unaffordable to implement in clinical setting. ${ }^{2,8}$ Markerless approaches with few cameras, namely Microsoft Kinect, are emerging techniques to study human motion. ${ }^{16}$ Nevertheless, the exact accuracy of these markerless systems is still unclear, ${ }^{17}$ namely in kinematic analysis of the poststroke patients ULs. Electromagnetic motion capture systems are another possible alternative due to their small size, high sampling rate and precision. ${ }^{18}$ They consist of a source that emits an electromagnetic field, which is used to determine the location and orientation of sensors. ${ }^{13}$ However, the presence of metals $^{19}$ and other electromagnetic sources such as cellphones, power lines, or other devices affects these systems and their correction is lengthy and complicated. ${ }^{13}$ Miniature Inertial Measurement Units are another emerging system ${ }^{16}$ which could be another option, due to their small size and portability. ${ }^{13}$ They can combine accelerometers, gyroscopes, and magnetometers, ${ }^{18}$ resulting in increased accuracy. ${ }^{13}$ Nevertheless, they may undergo electromagnetic interference as well and the degree of accuracy and reliability is site and task specific. ${ }^{13}$ The variety of available systems triggers the question: what type of system has been used to kinematically assess ULs in healthy and poststroke adults, in the last decade?

In addition, the identification of the most relevant kinematic metrics reflecting ULs motor impairment and functional deficits, as well as their interpretation and translation to clinically interpretable measures, requires clarification. 3,11,20,21 Many kinematic metrics have been used in the evaluation of UL movements in poststroke patients. ${ }^{22}$ Based on the theories of UL movement planning, ${ }^{23}$ these metrics can be classified into two categories: end-point (hand or wrist) kinematic metrics and joint kinematic metrics. ${ }^{12,23}$ End-point kinematic metrics are widely calculated by $3 \mathrm{D}$ Cartesian coordinates of only one marker on the wrist (or hand) and include linear metrics like peak velocity, movement smoothness, and movement straightness of the end-point displacement. ${ }^{12}$ Joint kinematic metrics include joint range of motion and inter-joint correlation (coordination). Trunk displacement has also been used to quantify compensatory strategies and may also be considered within joint kinematics. ${ }^{8}$ Subramanian et al. ${ }^{24}$ suggested the association between the end-point kinematics and the motor performance, as well as between the joint kinematics and the movement quality. However, this association and its meaning to stroke rehabilitation and research are not well established. Subramanian et al., ${ }^{24}$ and other authors, ${ }^{8}$ suggested also that movement quality kinematics are more sensitive in identifying UL deficits, while others ${ }^{6,11}$ have argued that motor performance kinematics are sensitive to change over time and discriminate healthy subjects from those with stroke, as well as subjects with moderate impairment from those with mild impairment. Murphy et al. ${ }^{20}$ speculate also that some metrics, like trunk displacement, reflect primarily the component of compensation, and others, like movement smoothness, the restitution. This type of association may be important to evaluate the intervention effect: compensation or restitution.

Based on the presented problems, the aim of this second part was to review and discuss the methods used to analyze the kinematics of ULs of healthy and poststroke adults, namely motion capture systems and kinematic extracted metrics.

\section{Methods}

The study was conducted using the systematic review method proposed by the Preferred Reporting Items for Systematic Reviews and Meta-Analysis. ${ }^{25}$

\subsection{Research questions}

The two major research questions of this review were:

(1) What are the motion capture systems used in literature that analyzed the kinematics of ULs in healthy and poststroke adults?

(2) What are the kinematic metrics extracted in these same articles?

\subsection{Search strategy}

Two reviewers performed an electronic search on PubMed database and the resource aggregator B-on, namely using the EBSCO EDS interface, to find all the articles published between 1 January 2007 and 31 December 2017 on the topic of UL kinematic analysis in healthy and poststroke adults. The following search terms combinations were used: ("upper extremity" OR "upper limb” OR arm) AND (kinematic OR motion OR movement) AND (analysis OR assessment OR measurement). The search terms were limited to titles of available full scientific papers, published in academic journals, and written in English. The reference lists of all articles were also scanned to identify other potential eligible articles.

\subsection{Inclusion and exclusion criteria}

The articles included in this review: (1) had the purpose to analyze objectively 3D kinematic of ULs; (2) studied clearly described functional movements of ULs, or ADL involving ULs (according to van Tuijl et al. $^{26}$ ); and (3) studied healthy living adult ( $>19$ years old) humans and/or adult humans with stroke sequelae. The articles excluded from this review: (1) analyzed a single UL joint rather than the UL itself, according to the SRRR recommendations; ${ }^{3}$ (2) studied athletes, to eliminate the sport gesture influence on the UL movement; (3) used robots, exoskeletons, or virtual realities, to study more realistic contexts; (4) were meta-analyses, reviews, case reports, pilot studies, technical notes, or studies published as conference proceedings.

\subsection{Assessment of methodologic quality}

The articles included in this systematic review were evaluated using a quality index proposed by Downs and Black. ${ }^{27}$ West et al. $^{28}$ identified the Downs and Black checklist as being consistent with 18 other recommended quality assessment systems. Studies meeting $<60 \%$ criteria were considered low quality, $\geq 60-<75 \%$ moderate quality, and $\geq 75 \%$ high quality. 
The two searching reviewers independently performed the quality assessment for each of the included articles. Consensus regarding the quality index score for each article was achieved by both authors.

\subsection{Data extraction}

Data from the included articles were extracted by one reviewer and then checked by a second reviewer using a data extraction table (Table 1) which identified: author identification, year of publication, motion capture systems, kinematic metrics, and quality index score.

\section{Results}

\subsection{Search yield}

The search strategy revealed 471 results and 3 other articles were identified through the reference lists (Table 2). After an initial examination, 329 were rejected as copies of the same paper; the remaining 145 articles were then reviewed by the two independent reviewers. From these, 86 were not included since they: (1) studied sport gestures, passive movements, purposeless or unclear movements and/or (2) examined children, animals, corpses, or other pathologic conditions. From the 59 included articles, 45 were excluded as they: (1) analyzed only one joint of the UL; (2) were in athletes; (3) used robots, exoskeletons, or virtual realities; and/or (4) were meta-analyses, reviews, case reports, pilot studies, technical notes, or studies published as conference proceedings.

A total of 14 articles were considered in the current review as shown in Figure 1, of which four included a healthy sample, ${ }^{21,29-31}$ three studied poststroke patients $\mathrm{s}^{20,22,32}$ and seven comprised both a stroke group and a healthy/control group., ${ }^{2,-11}$

\subsection{Motion capture systems}

The most widely used type of motion capture system, either in articles with healthy participants or in articles with poststroke participants, was the optoelectronic with passive markers, $7,9,11,20-22,31,32$ with a number of cameras ranging from $5^{11,20,32}$ to $12 .{ }^{7}$ Chen et al. ${ }^{21}$ were the only ones who did not identified the number of cameras used. Other two articles used other optoelectronic systems variations: one selected active LED-markers with three cameras ${ }^{29}$ and the other chose the Microsoft Kinect v2 with one camera. ${ }^{8}$

Other three articles ${ }^{6,10,30}$ used electromagnetic systems and only one article ${ }^{2}$ used an inertial system.

\subsection{Kinematic metrics}

Most articles analyzed both "joint kinematics" and "end-point kinematics;" ${ }^{\prime-11,20,22,31,32}$ two, ${ }^{2,6}$ involving poststroke adults, analyzed only "end-point kinematics;" and two, ${ }^{29,30}$ involving just healthy adults, analyzed only "joint kinematics." Chen et al. ${ }^{21}$ analyzed two variables related to robotic applications, which do not fit the above categorization: "dexterity measure" and "manipulability ellipsoid."
In descending order of use frequency, the analyzed "endpoint kinematics" were: movement duration; $;$, $6,7,9-11,20,31,32$ peak velocity; ${ }^{6-8,10,11,22}$ number of movement units (or velocity peaks); ${ }^{6,11,20,22,32}$ index of curvature (or reach path ratio); ${ }^{6-8,22}$ reach extent (or trajectory length); ${ }^{6,22,31}$ absolute and relative times for each phase; ${ }^{9,11}$ time and percentage of time to peak velocity; ${ }^{6,11}$ mean/peak velocity; ${ }^{6,10}$ end-point error; ${ }^{22}$ first velocity peak, time and percentage of time to first peak; ${ }^{11}$ and mean velocity. ${ }^{10}$

In descending order of use frequency, the analyzed "joint kinematics" were: joint angles of shoulder and elbow; $;^{9-11,29-31}$ trunk displacement; ${ }^{7,8,11,20,32}$ joint angles of wrist; ${ }^{9,29-31}$ range of motion of shoulder, elbow, ${ }^{9,22,29,31}$ and wrist; ${ }^{9,29,31}$ interjoint coordination between shoulder and elbow; ${ }^{8,11,22}$ peak angular velocity of elbow; ${ }^{11,32}$ angular joint motion for shoulder and elbow; ${ }^{11}$ angular velocities of shoulder and elbow; ${ }^{9}$ and maximum aperture and percentage of movement cycle where maximum aperture occurs. ${ }^{7}$

\section{Discussion}

In this second part of systematic review, we analyzed the same literature of the first on $e^{33}$ in order to identify which were (1) the motion capture systems that were being used in healthy and poststroke adults and (2) the kinematic metrics extracted in these same articles. In addition, this systematic review triggers a reflection on relevant elements to be considered in future studies.

\subsection{Motion capture systems}

First, most of the articles used optoelectronic systems (with passive markers), possibly because this type of system is more widespread, is accurate, and presents the best relation between the advantages and the limitations regarding its use, when comparing with other systems ${ }^{14,17}$ However, the laboratory and task-specific error assessments to guarantee the control of whole measurement process ${ }^{34}$ are missing in most of the reviewed studies, which can compromise their validity and comparison between them. Actually, the data of optoelectronic systems could suffer from a number of inaccuracy sources, collectively termed instrumental errors, ${ }^{16}$ due to the use of a camera-based approach which has been found to be dependent on: the number and position of the cameras, ${ }^{35,36}$ their lens distortion, ${ }^{37}$ the dimension of the capture volume, ${ }^{38}$ and the algorithms used for the reconstruction of a marker's 3D position. ${ }^{39}$ The number of cameras was the only referred factor to be mentioned by most authors, with the exception of the study of Chen et al. ${ }^{21}$ Position of the cameras was only referred in the study of Murphy et al. ${ }^{11}$ Eichelberger et al. $^{34}$ advocated that instrumental errors should also be determined and documented relative to various task-specific movement protocols to guarantee a highquality research. Therefore, according to these recommendations, ${ }^{34,35,38}$ future research should evaluate the system-specific error in the laboratory and for the task performed, presenting that data.

Because of the difficulty in transportation, large set-up volume, and high cost, optoelectronic systems hamper 
TOPICS IN STROKE REHABILITATION

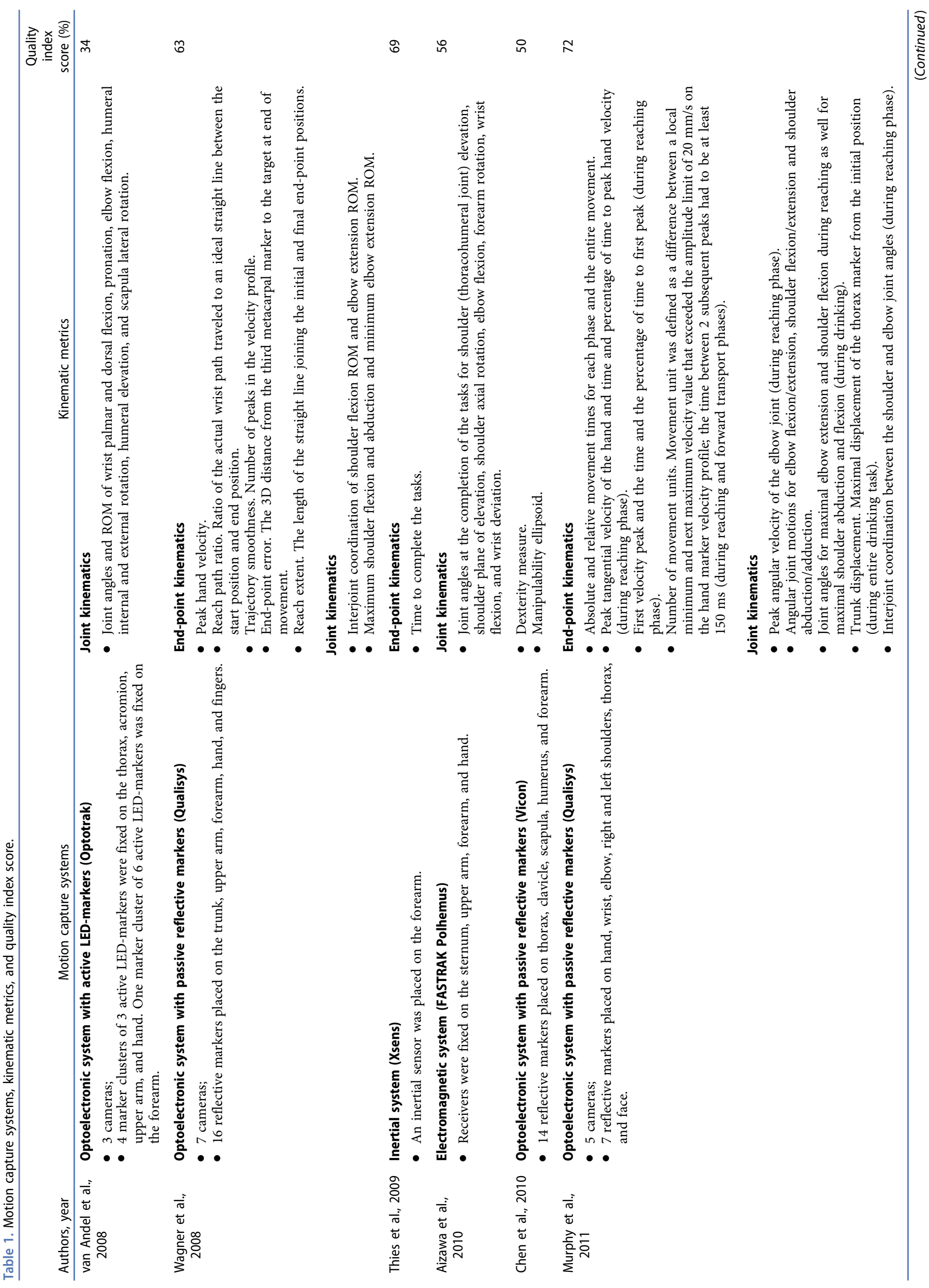




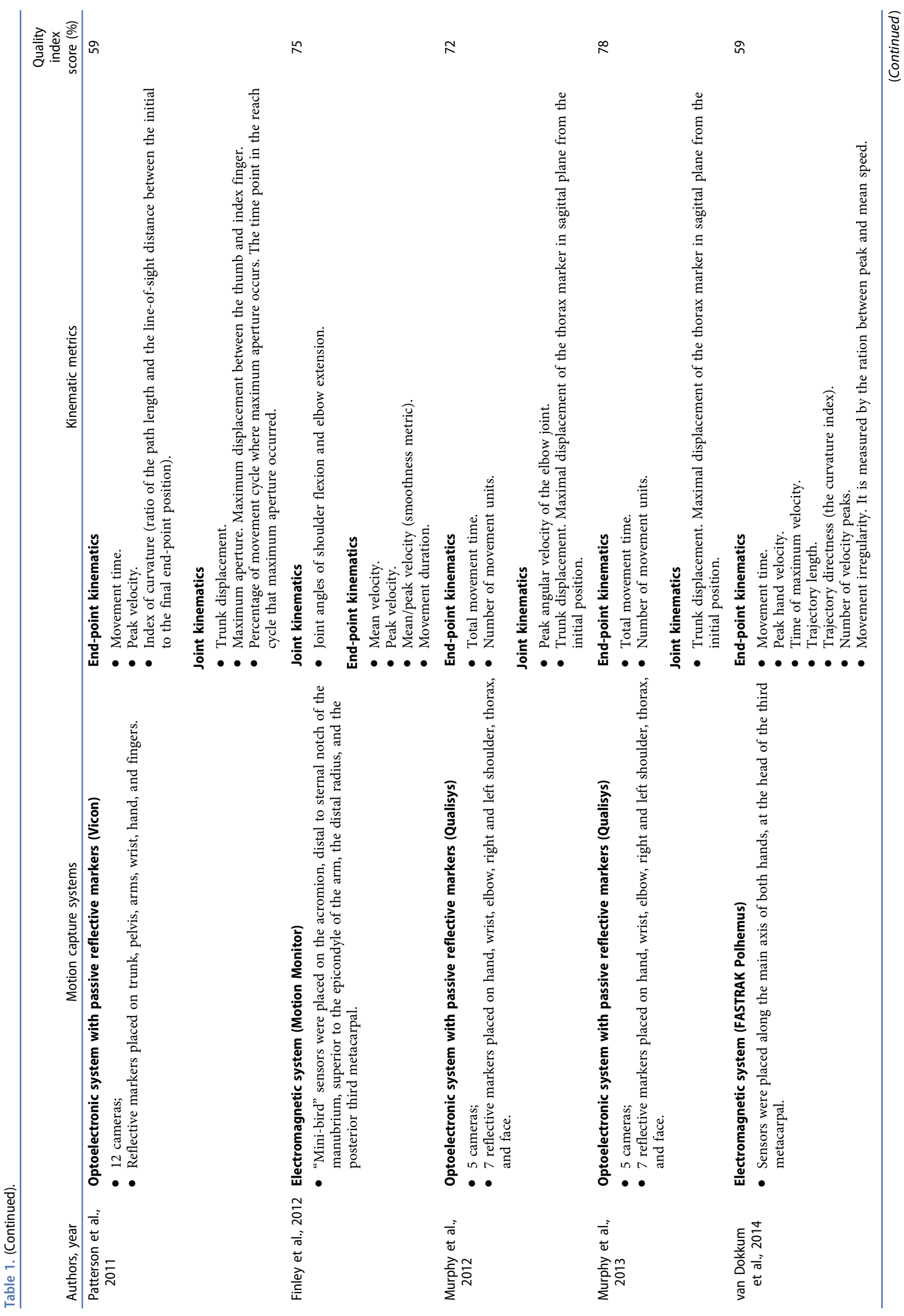




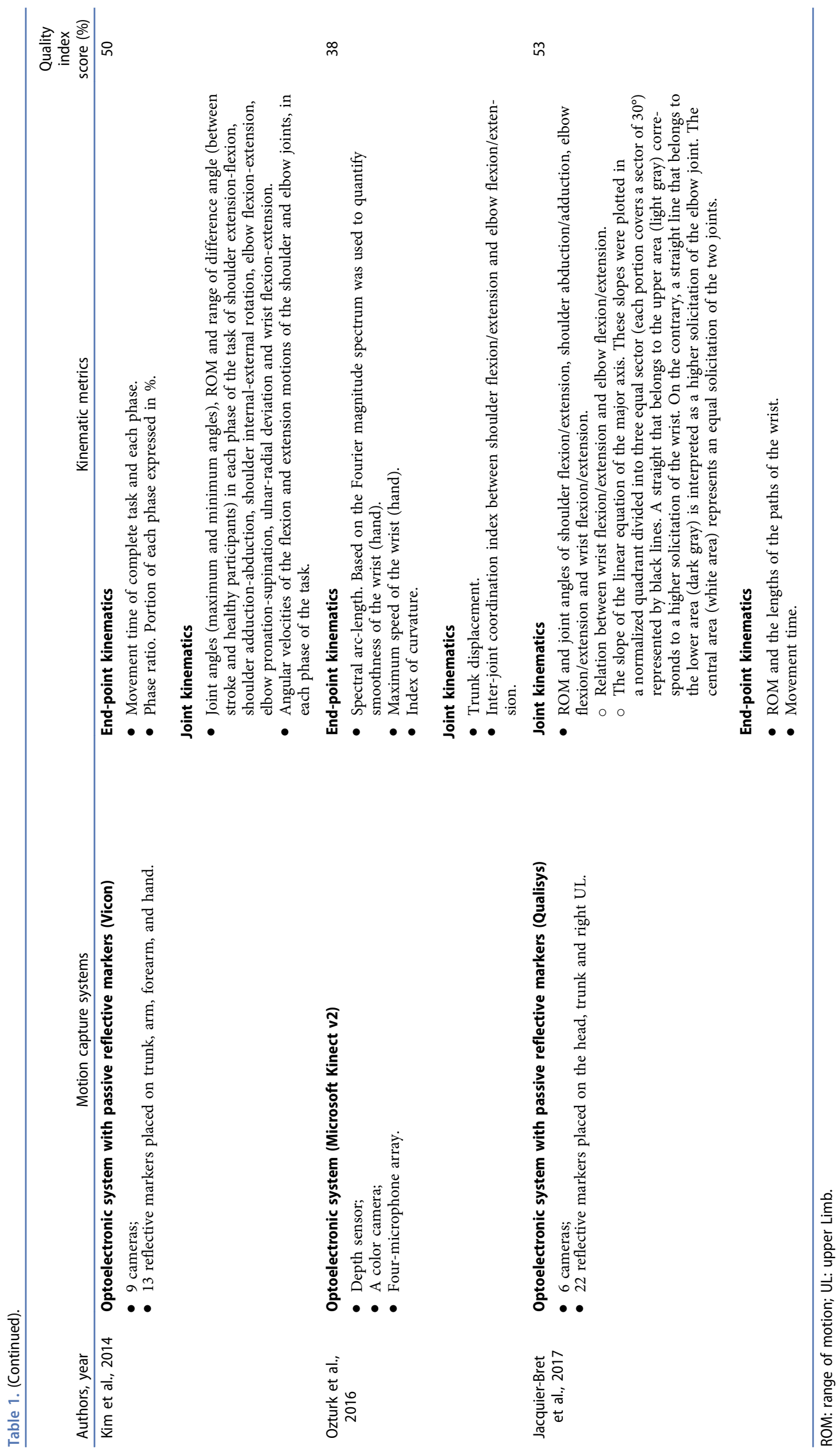


Table 2. Number of papers collected from PubMed and B-on.

\begin{tabular}{|c|c|c|c|}
\hline Search terms & PubMed & B-on & References \\
\hline $\begin{array}{l}\text { "upper extremity" OR } \\
\text { "upper limb" OR arm } \\
\text { AND } \\
\text { kinematics OR } \\
\text { motion OR movement } \\
\text { AND } \\
\text { analysis OR } \\
\text { assessment OR } \\
\text { measurement }\end{array}$ & 20 & 451 & 3 \\
\hline
\end{tabular}

evaluation of poststroke patients in acute and sub-acute phases, during hospitalization or at rehabilitation centers. ${ }^{2,8}$ In fact, only two articles (of Murphy et al.) involving this type of systems evaluated subjects in the acute phase ${ }^{20}$ and subacute $^{32}$ phases after stroke, respectively (please see the first part of the review ${ }^{33}$ for more details regarding sample characteristics). Other two articles analyzed poststroke adults in the acute and sub-acute phases using an electromagnetic system ${ }^{6}$ and the Microsoft Kinect, ${ }^{8}$ respectively. Just one study, ${ }^{2}$ which analyzed the chronic phase, used an inertial system. Although these portable systems appear to be promising alternatives for the kinematic analysis of the ULs in stroke patients, the literature proving its validity for this purpose is scarce $e^{13,19,40}$ and it is likely to benefit from reproducibility of outcome measures.

Therefore, in the coming years, it is emergent to focus on the development of accurate and reliable motion acquisition systems which do not encumber the performer or influence their natural movement and that can be easily transported and used in a hospital or other clinical context. These systems will allow the evaluation of more subjects in the different stages of poststroke rehabilitation and, consequently, they will contribute to a deeper understanding of the mechanisms underlying the motor recovery of the ULs after stroke.

\subsection{Kinematic metrics}

The authors of the articles under review analyzed several different linear and angular kinematic variables which may be related to the lack of clarity regarding the ULs motor planning, ${ }^{23}$ i.e. are ULs movements planned by joint angle and/or by end-point coordinates? Most authors analyzed "joint kinematics" and "end-point kinematics," of which "movement time," "peak velocity," "number of movement units (velocity peaks)," "joint angles of shoulder and elbow," and "trunk displacement" were the most studied. According to Reyes-Guzmán et al., ${ }^{12}$ these kinematic metrics quantify different characteristics of the UL movements: "movement time" and "peak velocity" are related with the speed; the "number of velocity peaks" measure the smoothness; the "joint angles of shoulder and elbow" translate the functional range of motion; and the "trunk displacement" shows compensation. Despite this, we should question if their analysis is sufficient to improve the understanding about the mechanisms driving motor recovery and to differentiate restitution from compensation. Furthermore, UL function includes reaching, grasping, moving, and manipulating objects in a great number of activities of daily living. ${ }^{23}$ The

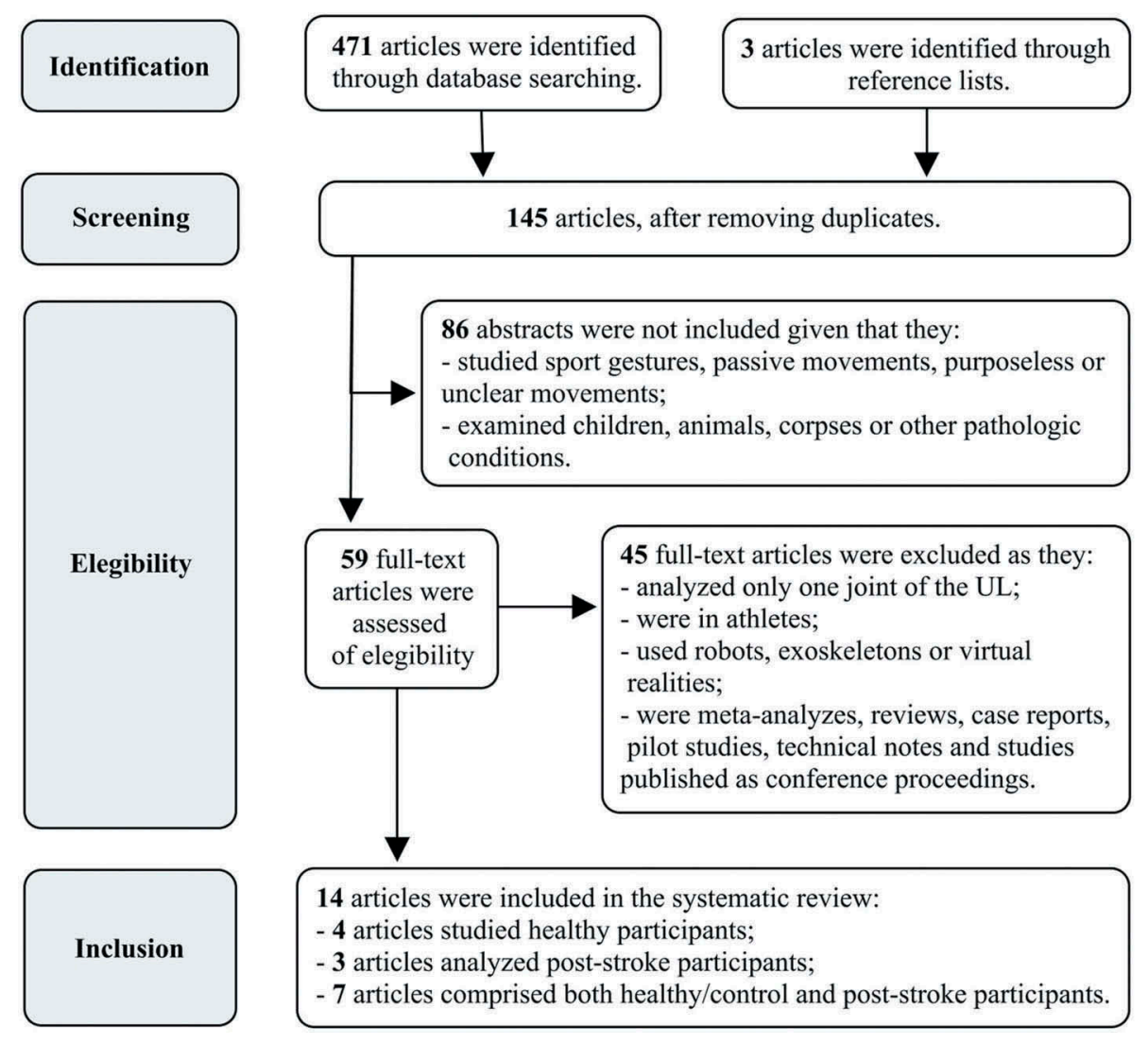

Figure 1. Review selection and exclusion criteria. 
abovementioned measurements are mainly associated with reaching, but they do not measure the abilities to open the hand, to grasp, to hold, and to move objects. Although many authors have defined as an inclusion criterion the ability to perform tasks involving these skills, such as drinking, they did not evaluate them in their studies. Patterson et al. ${ }^{7}$ were the only ones who analyzed index finger and thumb movements, namely maximum aperture and percentage of movement cycle where maximum aperture occurs. Without linear and angular data characterizing the ability to open and close the hand, the clinical utility of the current data appears to be very limited. Further studies should examine grasping and manipulation to ensure appropriate assessment, intervention, and patients' integration into the daily life.

In addition to the lack of clarity regarding the ULs motor planning, the variability in stroke extension and location ${ }^{33}$ increases the difficulty in the definition of the variables set to analyze. Depending on the injured area, the deficits may result from problems in target location, eye-hand coordination, temporal coordination, postural control, motor units recruitment, among others. ${ }^{23}$ Therefore, should kinematic variables set be defined without considering the stroke location and respective affected functions? Stroke describes a very heterogeneous group of clinical conditions that are unified by a vascular injury, but not by size, location, or impact of injury. ${ }^{41}$ Despite this, clinical trials are often designed with a "one size fits all" point of view. ${ }^{41}$ The articles included in this review analyzed the variables without considering the different stroke locations and studied the participants as a homogeneous sample, which can make them vulnerable to patient heterogeneity. Thus, to improve specific and effective neurorehabilitation strategies, it is crucial that future studies direct their attention to the influence of the stroke location on ULs' movement to allow a better understanding of the produced deficits. If the establishment of homogeneous groups regarding stroke location is not conceivable, case series and/ or case-control series should be considered as more appropriate studies to understand this question.

One last important issue is the paucity of information describing the psychometric properties (e.g. reliability, validity, and sensitivity to change) of kinematic metrics of UL. ${ }^{42,43}$ Only three articles ${ }^{7,20,22}$ described psychometric properties of kinematic assessment, namely the reliability ${ }^{7,22}$ and the responsiveness to external change. ${ }^{20}$ To establish a core set of kinematic outcomes, it is important that future studies describe their psychometric properties, either when they use kinematic variables as discriminative measures (to discriminate UL motor performance of people with stroke from that of people without stroke) or when they use them as evaluative measures (to evaluate longitudinal change in UL motor performance). ${ }^{22}$ For use as a discriminative measure, kinematic data must demonstrate construct validity and reliability based on stable between-subject variations. ${ }^{22}$ For use as an evaluative measure, kinematic data must demonstrate longitudinal construct validity, reliability based on stable withinsubject variations, and responsiveness (the ability to detect minimal clinically important change). ${ }^{22}$

In summary, the present systematic review identified the motion capture systems used and kinematic metrics extracted for ULs' kinematic analysis: most articles used optoelectronic systems, however, without presentation of laboratory-or taskspecific errors; and most authors analyzed "joint kinematics" and "end-point kinematics," mainly related with reaching. Markerless systems, used in some studies, seem to be promising alternatives for implementation of kinematic analysis in hospitals and clinics, but the literature proving their validity is scarce. The different stroke locations of participants were not considered in the analysis of kinematic metrics and only three articles described their psychometric properties. Therefore, some gaps were identified in most of the articles analyzed, which may compromise the creation of valid databases of ULs kinematics. To avoid these problems, future research should: (1) validate the emergent portable motion capture systems to kinematic assessment of ULs; (2) document the specific error of the motion capture systems at the acquisition place and for the studied task; (3) include grasping and manipulation analysis; (4) study the influence of the stroke location on ULs kinematic metrics; and (5) describe their psychometric properties.

\section{ORCID}

Inês Albuquerque Mesquita (D) http://orcid.org/0000-0001-9659-7531 Pedro Filipe Pereira da Fonseca (D) http://orcid.org/0000-0002-48854924

Ana Rita Vieira Pinheiro (D) http://orcid.org/0000-0003-4310-7652

Miguel Fernando Paiva Velhote Correia (D) http://orcid.org/0000-00016065-9358

Cláudia Isabel Costa da Silva (iD http://orcid.org/0000-0002-1484-1893

\section{References}

1. Cramer SC, Nelles G, Benson RR, et al. A functional MRI study of subjects recovered from hemiparetic stroke. Stroke. 1997;28 (12):2518-2527.

2. Thies SB, Tresadern PA, Kenney LP, et al. Movement variability in stroke patients and controls performing two upper limb functional tasks: a new assessment methodology. J Neuroeng Rehabil. 2009;6 (2). doi:10.1186/1743-0003-6-2.

3. Kwakkel G, Lannin NA, Borschmann K, et al. Standardized measurement of sensorimotor recovery in stroke trials: consensus-based core recommendations from the Stroke Recovery and Rehabilitation Roundtable. Int J Stroke. 2017;12(5):451-461. doi:10.1177/1747493017711813.

4. Fugl-Meyer AR, Jääskö L, Leyman I, Olsson S, Steglind S. The post-stroke hemiplegic patient. 1. a method for evaluation of physical performance. Scand J Rehabil Med. 1975;7:13-31.

5. Lyle RC. A performance test for assessment of upper limb function in physical rehabilitation treatment and research. Int J Rehabil Res. 1981;4:483-492.

6. van Dokkum L, Hauret I, Mottet D, Froger J, Métrot J, Laffont I. The contribution of kinematics in the assessment of upper limb motor recovery early after stroke. Neurorehabil Neural Repair. 2014;28(1):4-12. doi:10.1177/1545968313498514.

7. Patterson TS, Bishop MD, McGuirk TE, Sethi A, Richards LG. Reliability of upper extremity kinematics while performing different tasks in individuals with stroke. J Mot Behav. 2011;43 (2):121-130. doi:10.1080/00222895.2010.548422.

8. Ozturk A, Tartar A, Ersoz Huseyinsinoglu B, Ertas AH. A clinically feasible kinematic assessment method of upper extremity motor function impairment after stroke. Measurement. 2016;80 (SupplementC):207-216. doi:10.1016/j.measurement.2015.11.026.

9. Kim K, Song WK, Lee J, et al. Kinematic analysis of upper extremity movement during drinking in hemiplegic subjects. Clin Biomech. 2014;29(3):248-256. doi:10.1016/j.clinbiomech.2013.12.013. 
10. Finley M, Combs S, Carnahan K, Peacock S, Van Buskirk A. Comparison of "less affected limb" reaching kinematics in individuals with chronic stroke and healthy age-matched controls. Phys Occup Ther Geriatrics. 2012;30(3):245-259. doi:10.3109/02703181.2012.716506.

11. Murphy MA, Will_En KS, Sunnerhagen KS. Kinematic variables quantifying upper extremity performance after stroke during reaching and drinking from a glass. Neurorehabil Neural Repair. 2011;25(1):71-80. doi:10.1177/1545968310370748.

12. de Los Reyes-Guzmán A, Dimbwadyo-Terrer I, Trincado-Alonso F, Monasterio-Huelin F, Torricelli D, Gil-Agudo A. Quantitative assessment based on kinematic measures of functional impairments during upper extremity movements: a review. Clin Biomech. 2014;29 (7):719-727. doi:10.1016/j.clinbiomech.2014.06.013.

13. Cuesta-Vargas AI, Galan-Mercant A, Williams JM. The use of inertial sensors system for human motion analysis. Phys Ther Rev. 2010;15(6):462-473. doi:10.1179/1743288X11Y.0000000006.

14. Vilas-Boas Mdo C, Cunha JP. Movement quantification in neurological diseases: methods and applications. IEEE Rev Biomed Eng. 2016;9:15-31. doi:10.1109/RBME.2016.2543683.

15. Domingues A, Barbosa F, Pereira EM, et al. Towards a detailed anthropometric body characterization using the Microsoft Kinect. Technol Health Care. 2016;24(2):251-265. doi:10.3233/THC-151116.

16. Di Marco R, Rossi S, Castelli E, Patanè F, Mazzà C, Cappa P. Effects of the calibration procedure on the metrological performances of stereophotogrammetric systems for human movement analysis. Measurement. 2017;101:265-271. doi:10.1016/j. measurement.2016.01.008.

17. Colyer SL, Evans M, Cosker DP, Salo AIT. A review of the evolution of vision-based motion analysis and the integration of advanced computer vision methods towards developing a markerless system. Sports Med Open. 2018;4(1):24. doi:10.1186/ s40798-018-0138-z.

18. Pérez R, Costa Ú, Torrent M, et al. Upper limb portable motion analysis system based on inertial technology for neurorehabilitation purposes. Sensors. 2010;10:12. doi:10.3390/s101210733.

19. Milne AD, Chess DG, Johnson JA, King GJ. Accuracy of an electromagnetic tracking device: a study of the optimal range and metal interference. J Biomech. 1996;29(6):791-793.

20. Murphy MA, Will_en C, Sunnerhagen KS. Responsiveness of upper extremity kinematic measures and clinical improvement during the first three months after stroke. Neurorehabil Neural Repair. 2013;27:844-853. doi:10.1177/1545968313491008.

21. Chen W, Xiong C, Huang X, Sun R, Xiong Y. Kinematic analysis and dexterity evaluation of upper extremity in activities of daily living. Gait Posture. 2010;32(4):475-481. doi:10.1016/j.gaitpost.2010.07.005.

22. Wagner JM, Rhodes JA, Patten C. Reproducibility and minimal detectable change of three-dimensional kinematic analysis of reaching tasks in people with hemiparesis after stroke. Phys Ther. 2008;88(5):652-663. doi:10.2522/ptj.20070255.

23. Shumway-Cook A, Woollacott MH. Motor Control: Translating Research into Clinical Practice. 5th ed. Philadelphia, PA; London: Lippincott Williams \& Wilkins; 2017.

24. Subramanian SK, Yamanaka J, Chilingaryan G, Levin MF. Validity of movement pattern kinematics as measures of arm motor impairment poststroke. Stroke. 2010;41(10):2303. doi:10.1161/STROKEAHA.109.572750.

25. Moher D, Liberati A, Tetzlaff J, Altman DG, Group P. Preferred reporting items for systematic reviews and meta-analyses: the PRISMA statement. Ann Intern Med. 2009;151:264-269, W264.

26. van Tuijl JH, Janssen-Potten YJ, Seelen HA. Evaluation of upper extremity motor function tests in tetraplegics. Spinal Cord. 2002;40(2):51-64.
27. Downs SH, Black N. The feasibility of creating a checklist for the assessment of the methodological quality both of randomised and non-randomised studies of health care interventions. J Epidemiol Community Health. 1998;52:377-384.

28. West S, King V, Carey TS, et al. Systems to Rate the Strength of Scientific Evidence. Rockville, MD: Agency for Healthcare Research and Quality: AHRQ Publication No. 02-E016; 2002.

29. van Andel CJ, Wolterbeek N, Doorenbosch CA, Veeger DH, Harlaar J. Complete 3D kinematics of upper extremity functional tasks. Gait Posture. 2008;27(1):120-127. doi:10.1016/j.gaitpost.2007.03.002.

30. Aizawa J, Masuda T, Koyama T, et al. Three-dimensional motion of the upper extremity joints during various activities of daily living. J Biomech. 2010;43(15):2915-2922. doi:10.1016/j.jbiomech.2010.07.006.

31. Jacquier-Bret J, Gorce P, Motti Lilian G, Vigouroux N. Biomechanical analysis of upper limb during the use of touch screen: motion strategies identification. Ergonomics. 2017;60 (3):358-365. doi:10.1080/00140139.2016.1175671.

32. Murphy MA, Willén C, Sunnerhagen KS. Movement kinematics during drinking task are associated with the activity capacity level after stroke. Neurorehabil Neural Repair. 2012;26(9):1106-1115. doi:10.1177/1545968312448234.

33. Mesquita IA, Pinheiro ARV, Velhote Correia MFP, Silva C. Methodological considerations for kinematic analysis of upper limbs in healthy and poststroke adults. Part I: a systematic review of sampling and motor tasks. Top Stroke Rehabil. 2019;26 (2): 142-152

34. Eichelberger P, Ferraro M, Minder U, et al. Analysis of accuracy in optical motion capture - A protocol for laboratory setup evaluation. J Biomech. 2016;49(10):2085-2088. doi:10.1016/j. jbiomech.2016.05.007.

35. Windolf M, Gotzen N, Morlock M. Systematic accuracy and precision analysis of video motion capturing systems-exemplified on the Vicon-460 system. J Biomech. 2008;41(12):2776-2780. doi:10.1016/j.jbiomech.2008.06.024.

36. Marco RD, Rossi S, Patanè F, Cappa P. Technical quality assessment of an optoelectronic system for movement analysis. J Phys Conf Ser. 2015;588(1):012030.

37. Weng J, Cohen P, Herniou M. Camera calibration with distortion models and accuracy evaluation. IEEE Trans Pattern Anal Mach Intell. 1992;14(10):965-980. doi:10.1109/34.159901.

38. Vander Linden DW, Carlson SJ, Hubbard RL. Reproducibility and accuracy of angle measurements obtained under static conditions with the Motion Analysis video system. Phys Ther. 1992;72 (4):300-305.

39. Abdel-Aziz YI, Karara HM, Hauck M. Direct linear transformation from comparator coordinates into object space coordinates in close-range photogrammetry ${ }^{\star}$. Photogramm Eng Remote Sensing. 2015;81(2):103-107. doi:10.14358/PERS.81.2.103.

40. Webster D, Celik O. Systematic review of Kinect applications in elderly care and stroke rehabilitation. J Neuroeng Rehabil. 2014;11 (1):108. doi:10.1186/1743-0003-11-108.

41. Boyd LA, Hayward KS, Ward NS, et al. Biomarkers of stroke recovery: consensus-based core recommendations from the stroke recovery and rehabilitation roundtable. Int J Stroke. 2017;12 (5):480-493. doi:10.1177/1747493017714176.

42. Roby-Brami A, Feydy A, Combeaud M, Biryukova EV, Bussel B, Levin MF. Motor compensation and recovery for reaching in stroke patients. Acta Neurol Scand. 2003;107(5):369-381.

43. Barak S, Duncan PW. Issues in selecting outcome measures to assess functional recovery after stroke. NeuroRX. 2006;3(4):505-524. doi:10.1016/j.nurx.2006.07.009. 